\title{
Tratamiento Fisioterapéutico en Caninos Domésticos con Claudicación del Tren Posterior
}

\author{
Physiotherapeutic Treatment in Domestic Dogs with Lameness of the Hind Limbs \\ Marelsy Pilco P. ${ }^{1}$, Elizabeth Hinostroza M.1, Enrique Serrano-Martínez ${ }^{1,2}$
}

\section{Resumen}

El estudio tuvo por objetivo evaluar el efecto de un tratamiento de fisioterapia (masajes, termoterapia, electroterapia y ultrasonido) en la recuperación de pacientes caninos con lesiones del tren posterior. Los canes de los 15 casos evaluados fueron de ambos sexos, con edades desde 5 meses hasta 12 años, que acudieron a la consulta veterinaria con claudicación del tren posterior por displasia de cadera, espondiloartrosis y neuropatías traumáticas. Los caninos fueron tratados hasta un máximo de 12 sesiones, donde las primeras seis sesiones eran dos veces por semana y las otras seis eran una vez por semana. Doce de los 15 pacientes se recuperaron de manera favorable y tres pacientes gerontes no respondieron al tratamiento. La aplicación de la fisioterapia tuvo una efectividad de $80 \%$ en caninos con problemas del tren posterior.

Palabras clave: fisioterapia; electroterapia; ultrasonido; canino

\section{AbSTRACT}

The objective of the study was to evaluate the effect of a physiotherapy treatment (massage, thermotherapy, electrotherapy and ultrasound) in the recovery of canine patients with hind limb injuries. The $\operatorname{dogs}(n=15)$ were of both sexes, with ages ranging from 5 months to 12 years, who attended the veterinary consultation with claudication of the hind limbs due to hip dysplasia, spondylarthrosis and traumatic neuropathies. The animals were treated for up to a maximum of 12 sessions, where the first six sessions were

\footnotetext{
${ }^{1}$ Clínica Veterinaria, Facultad de Medicina Veterinaria y Zootecnia, Universidad Peruana Cayetano Heredia, Lima, Perú

${ }^{2}$ E-mail: enrique.serrano@upch.pe
}

Recibido: 15 de noviembre de 2016

Aceptado para publicación: 14 de junio de 2017 
twice a week and the other six were once a week. Twelve of the 15 patients recovered favorably and three elderly patients did not respond to the treatment. The application of physiotherapy had an $80 \%$ effectiveness in canines with hind limbs problems.

Key words: physical therapy; electrotherapy; ultrasound; canine

\section{INTRODUCCIÓN}

La fisioterapia veterinaria es una rama de las ciencias de la salud que promueve alternativas terapéuticas de prevención, paliación y recuperación de alteraciones físicas; además, reduce el uso de medicamentos, mejorando y prolongando la calidad de vida de los pacientes (Del Pueyo, 2011). De acuerdo a la historia, los griegos y los romanos la usaban para corregir deformaciones torácicas y ciertas dolencias de los gladiadores; posterior a la Primera Guerra Mundial, la fisioterapia logró desarrollarse en modelos caninos, y ya en 1960 fue un tratamiento eficaz en equinos cuando se desarrolló el deporte de equitación (Jaramillo-Antillón, 2005; Van Dyke, 2009). Entre 1980 y 1986, en países como Reino Unido, Alemania, Suecia, Holanda y EEUU, surgió la rehabilitación canina dando inicio al Simposio de Medicina Internacional de Caninos Deportistas (McGonagle et al., 2004; Millis y Levine, 2013).

Los caninos con problemas neurológicos responden de manera favorable a la fisioterapia y es un buen paliativo para tratar la displasia de cadera y la osteoartrosis femoral (Oliver et al., 2003; Nelson y Couto, 2010). Las neuropatologías del tren posterior en caninos están ligadas con la degeneración discal, compresión de la medula espinal, ya sea de tipo adquirida o congénita (Nelson y Couto, 2010). La displasia de cadera es de carácter hereditario, frecuente en perros de raza grande y mediana, produce inestabilidad de la articulación coxofemoral, con signos de dolor, inflamación y paresia, conllevando a cambios degenerativos como osteoartrosis coxofemoral (Dávila et al., 2002; Nelson y Couto, 2010).

Los masajes durante 5 a 10 minutos (Del Pueyo, 2011; Ortega, 2013) reducen la tensión muscular por la acción de los receptores nerviosos cutáneos, al disminuir la secreción de ACTH, y en pacientes jóvenes favorece el desarrollo inmune (Del Pueyo, 2011). La aplicación del frío (bolsas de gel o hielo) a nivel local por 15 a 20 minutos, controla la inflamación y produce analgesia (MiróRodríguez et al., 2007).

La aplicación de calor superficial de 40$45{ }^{\circ} \mathrm{C}$ (de 15 a 30 minutos) tiene acción analgésica, produce vasodilatación, mejora el aporte de oxígeno y nutrientes, y reduce la rigidez articular y espasmos musculares, aunque está contraindicado en pacientes con inflamación aguda, edemas o hemorragias (Hury, 2008; Del Pueyo, 2011). La Electroestimulación Nerviosa Transcutánea o TENS estimula las fibras nerviosas periféricas y libera endorfinas de la asta ventral de la médula espinal produciendo analgesia (Correa, 2006). El ultrasonido es otra herramienta utilizado en la fisioterapia canina (Levine et al., 2001; Hanks et al., 2015).

El presente estudio tuvo por objetivo evaluar el efecto de un tratamiento de fisioterapia (masajes, termoterapia, electroterapia y ultrasonido) en la recuperación de los pacientes caninos con lesiones del tren posterior. 


\section{Materiales y Métodos}

\section{Lugar de Estudio}

El estudio se realizó con pacientes que acudieron a la Clínica Veterinaria Cayetano Heredia de la Facultad de Medicina Veterinaria y Zootecnia de la Universidad Peruana Cayetano Heredia, ubicada en el distrito de San Martín de Porres, Lima (Perú), entre agosto de 2015 a enero de 2016.

\section{Animales}

Se evaluaron 15 casos de caninos machos y hembras (excluyendo pacientes con problemas de piel, cardiópatas y hembras preñadas), con edades comprendidas entre cinco meses y doce años. Todos habían sido medicados con tratamientos convencionales (Cuadro 1).

Los pacientes contaban con registros completos de historias clínicas, radiografía, así como de mielografía o tomografía con diagnóstico de hernias lumbares, espondiloartrosis lumbar, displasia de cadera o poscirugía por traumatismo. Asimismo, se determinó el nivel inicial de dolor (leve [1-2], moderado [3-4], severo [5]), según las especificaciones de Colorado State University (2006), como se detalla:

- Score 0. Comportamiento feliz, enérgico, sensible, busca atención. Descansa bien, se para y camina normal en las cuatro patas. Se para, camina normal y rigidez leve al caminar. Mínima tensión corporal, no le afecta que lo toquen y no reacciona a la palpación de la articulación.

- $\quad$ Score 1. Comportamiento nervioso, un poco inquieto y agitado. Camina normal y rigidez leve al caminar. Tensión corporal leve, no le afecta que lo toquen excepto en la zona dolorida. Voltea la cabeza reconociendo la articulación dolorida.
- Score 2. Comportamiento ansioso, presenta brillo en los ojos. Durante la palpación presenta rigidez moderada al caminar. Leve tensión corporal, tira las extremidades durante la palpación de la articulación afectada.

- Score 3. Comportamiento ansioso, agresivo. Evita la interacción con las personas, se lame la zona dolorida. Postura anormal al ponerse de pie, no usa el área dolorosa al caminar. Tensión corporal moderada, responde agresivamente a la palpación de la articulación afectada.

- Score 4. Comportamiento deprimido, no responde al entorno, difícil distraerlo por el dolor. No soporta el peso sobre la extremidad, parece incómodo en reposo. No permite la palpación de la articulación. Es importante un plan analgésico.

Se programaron 12 sesiones fisioterapéuticas. Se inició con masajes (para reducir la ansiedad y el estrés), luego se aplicó compresas frías y calientes (efecto antinflamatorio), se siguió con electroterapia (efecto analgésico y para mejorar la tonicidad muscular), y se finalizó con el uso del ultrasonido (mejora el metabolismo local, favorece la síntesis de proteínas y la cicatrización), según Levine et al. (2001). Cada sesión tuvo una duración de 61 a 64 minutos. El tratamiento se llevó a cabo inicialmente con seis sesiones dos veces por semana y se continuó con una vez por semana hasta la recuperación del paciente, no siendo mayor a 12 sesiones programadas para este estudio; asimismo, los pacientes fueron auto-testigos. Los dueños de los canes indicaron por escrito en cuestionarios ad-hoc los avances positivos o negativos logrados después de la terapia.

\section{Tratamiento Fisioterapéutico}

Masajes. En las primeras dos sesiones, todo paciente recibió masajes relajantes (tipo stroking, en dirección al sentido del pelo y tipo Kneading, agarrando suavemente la porción muscular y tejido subcutáneo) de tres a cinco minutos con aceite aromático de ber- 
gamota (Citrus bergamia) de uso humano (Chándrika, Perú). Esta técnica fue empleada para aliviar el estrés o ansiedad del paciente (Lindley, 2015) y preparar la zona para efectuar el tratamiento fisioterapéutico.

Termoterapia y crioterapia. En las dos primeras sesiones se aplicó crioterapia mediante compresas de gel congelado a $0{ }^{\circ} \mathrm{C}$ (Nexcare 3M) durante 10 minutos. A partir de la tercera sesión, se incluyeron compresas de gel caliente de $40-43{ }^{\circ} \mathrm{C}$, luego de la crioterapia, durante 20 minutos. En ambos casos, la piel fue protegida con una tela delgada para evitar quemaduras. Se aplicaron compresas frías y calientes para aliviar el dolor, disminuir la inflamación y mejorar el aporte de oxígeno y nutrientes; además de reducir la rigidez articular y los espasmos musculares (Capote et al., 2009).

Electroestimulación Nerviosa Transcutánea (TENS). Se aplicó desde la primera sesión mediante la colocación de electrodos en la piel usando corriente eléctrica con una frecuencia de $1 \mathrm{a} 4 \mathrm{~Hz}$, de acuerdo a la sensibilidad del paciente, con una duración de 20 minutos. Se empleó un equipo de TENS (Globus MY STIM 2) y los programas $50 \mathrm{y}$ 52 para analgesia y electro-estimulación, respectivamente. TENS estimula las fibras nerviosas periféricas y libera endorfinas del asta ventral de la médula espinal, produciendo analgesia (Shearer y August, 2017); además, produce contracción muscular sin movilizar la articulación, teniendo en cuenta el ciclo terapéutico (1-15 miliamperios - $\mathrm{mA}$ ) para obtener sensación de hormigueo y contracción muscular del paciente (Correa, 2006; Capote et al., 2009).

Ultrasonido. En las dos primeras sesiones se aplicó ultrasonido con ciclos de trabajo pulsátil y continuo con $3.3 \mathrm{Mhz}$ (Levine et al. 2001; Hanks et al., 2015) de profundidad a nivel toracolumbar y lumbosacro, delimitando la zona por las apófisis vertebrales y aplicando solo a nivel muscular en el lado derecho e izquierdo. A partir de la tercera sesión se aplicó un ciclo de trabajo continuo. En ambos casos se hizo durante nueve minutos. Se empleó un equipo de medicina estética facial (RU-638 Supersonic).

\section{Análisis de Datos}

La efectividad fue determinada mediante la proporción de perros recuperados con la fisioterapia, según Kassai (1998), donde se considera «Muy efectivo» (>98\%), «Efectivo» (90-98\%), «Moderadamente efectivo» (80 a $89 \%)$, e «Insuficientemente efectivo» $(<80 \%)$.

\section{Resultados}

Los casos más frecuentes fueron de displasia de cadera $(26.7 \%)$ y espondiloartrosis lumbar (26.6\%) (Cuadro 1).

El resultado del empleo de las técnicas de masajes, termoterapia, electroterapia y ultrasonido (Figura 1), en los 15 pacientes con afecciones neurológicas y neuropatías traumáticas en caninos domésticos se presenta en los cuadros 2 y 3 . La respuesta positiva al efecto analgésico y antiinflamatorio del tratamiento se presentó en cinco pacientes en la segunda sesión, en tres en la tercera sesión, en tres en la cuarta sesión y en un paciente en la quinta sesión (Cuadro 2). En general, el tratamiento resultó efectivo en el $80 \%(12 / 15)$ de los pacientes (Cuadro 3$)$.

\section{Discusión}

El estudio demostró que el uso de la terapia física en caninos con problemas del tren posterior llegó a tener a tener una efectividad del $80 \%(12 / 15)$ de acuerdo a la escala de Kassai (1998), lo cual puede ser considerado como una efectividad moderada en el tratamiento fisioterapéutico. Los resultados refuerzan lo indicado por Nelson y Couto (2010), quienes manifiestan que los pacientes con neuropatías traumáticas focales responden a la fisioterapia con calor y masajes, 
Cuadro 1. Características de los caninos previo a la ejecución de la terapia física

\begin{tabular}{|c|c|c|c|c|c|c|}
\hline Paciente & Raza & Sexo & $\begin{array}{l}\text { Edad } \\
\text { (años) }\end{array}$ & Diagnóstico clínico & $\begin{array}{l}\text { Score de } \\
\text { dolor } \\
\text { inicial }^{\text {(A) }}\end{array}$ & Medicación \\
\hline 1 & $\begin{array}{l}\text { Bulldog } \\
\text { Inglés }\end{array}$ & $\mathrm{M}$ & 0.5 & $\begin{array}{l}\text { Displasia cadera } \mathrm{MP}\left[\operatorname{LD} 87^{\circ} \text { y }\right. \\
\left.\text { LI } 100^{\circ}\right]\end{array}$ & $3-4$ & Sí (1) (2) \\
\hline 2 & $\begin{array}{l}\text { Golden } \\
\text { Retriever }\end{array}$ & $\mathrm{H}$ & 0.7 & Displasia cadera MPD, $96^{\circ}$ & $1-2$ & Sí (2) (3) \\
\hline 3 & Pequinés & M & 1 & $\begin{array}{l}\text { Poscirugía ( } 1 \text { mes }) \text { por } \\
\text { politraumatismo }\end{array}$ & $3-4$ & No \\
\hline 4 & Mestizo & M & 1 & $\begin{array}{l}\text { Post-cirugía femoral por } \\
\text { traumatismo }\end{array}$ & $3-4$ & Sí (1) (4) \\
\hline 5 & Cocker & $\mathrm{H}$ & 4 & $\begin{array}{l}\text { Estenosis vertebral L7 -S1y } \\
\text { cauda equina }\end{array}$ & $3-4$ & Sí (5) \\
\hline 6 & Rottweiler & M & 5 & $\begin{array}{l}\text { Displasia cadera }\left[\mathrm{LI} 96^{\circ} \text { y }\right. \\
\text { hernia lumbar L7 - S1 }\end{array}$ & $1-2$ & Sí (1) (2) \\
\hline 7 & Bull terrier & M & 5 & Displasia de cadera MPI, $100^{\circ}$ & $1-2$ & $\mathrm{Sí}^{(6)}$ \\
\hline 8 & Collie & $\mathrm{H}$ & 5 & $\begin{array}{l}\text { Espondiloartrosis T5-T6, T10- } \\
\text { T11 }\end{array}$ & $1-2$ & Sí (2) (3) \\
\hline 9 & Cruzado & M & 6 & Hernia protrusiva lumbar & $1-2$ & Sí (1) (2) \\
\hline 10 & Pekinés & $\mathrm{H}$ & 7 & $\begin{array}{l}\text { Cirugía hemilaminectomía (1 } \\
\text { mes). Condrodistrofia T10, } \\
\text { T11 }\end{array}$ & $3-4$ & No \\
\hline 11 & Mestizo & $\mathrm{H}$ & 9 & $\begin{array}{l}\text { Síndrome cauda equina y } \\
\text { artrosis de rodilla }\end{array}$ & $1-2$ & $\mathrm{Si}^{\prime}{ }^{(1)(2)}$ \\
\hline 12 & $\begin{array}{l}\text { Ovejero } \\
\text { Inglés }\end{array}$ & $\mathrm{H}$ & 9 & $\begin{array}{l}\text { Espondiloartrosis vertebral L7 } \\
- \text { S1 }\end{array}$ & $1-2$ & $\mathrm{Si}^{\prime}{ }^{(1)(2)}$ \\
\hline 13 & Mestizo & M & 9 & $\begin{array}{l}\text { Espondiloartrosis deformante } \\
\text { L7 S1, parálisis flácida MP(C) }\end{array}$ & 5 & $\mathrm{Sí}^{\prime}{ }^{(2)}(5)$ \\
\hline 14 & $\begin{array}{l}\text { Setter } \\
\text { Irlandés }\end{array}$ & $\mathrm{M}$ & 12 & $\begin{array}{l}\text { Espondiloartrosis lumbar L7- } \\
\text { S1, Cauda equina }\end{array}$ & $1-2$ & $\mathrm{Sí}^{(4)}$ \\
\hline 15 & Siberiano & $\mathrm{H}$ & 12 & $\begin{array}{l}\text { Artrosis coxofemoral derecha } \\
\text { moderada }\end{array}$ & $3-4$ & $\mathrm{Sí}^{\prime}{ }^{(2)(6)}$ \\
\hline
\end{tabular}

A Colorado State University (2006)

MP: Miembro posterior; LD: Lado derecho; LI: Lado izquierdo, MPD: Miembro posterior derecho; MPI: Miembro posterior izquierdo

B Tumor mamario y lipoma

C Infección urinaria y neoplasia testicular

${ }^{1}$ AINE (Meloxivet $0.1 \mathrm{mg} / \mathrm{kg} \times 6 \mathrm{~d}$ )

${ }^{2}$ Tramadol 3 o $4 \mathrm{mg} / \mathrm{kg} \times 5 \mathrm{~d}$

${ }^{3}$ AINE (Meloxicam $0.25 \mathrm{mg} / \mathrm{kg} \times 6 \mathrm{~d}$ )

${ }^{4}$ Vitaminas (Osteocart plus)

${ }^{5}$ Glucocorticoide (Metilprednisolona $10 \mathrm{mg} / \mathrm{kg}$ x $10 \mathrm{~d}$ )

${ }^{6}$ AINE (Rimadyl $100 \mathrm{mg} 1 / 2$ tableta c/12 horas $\times 8$ a $10 \mathrm{~d}$ ) 
Cuadro 2. Características y estado de recuperación de caninos con afecciones neurológicas y neuropatías traumáticas que fueron sometidos a terapia física

\begin{tabular}{|c|c|c|c|c|c|}
\hline Paciente & $\begin{array}{l}\text { Edad } \\
\text { (años) }\end{array}$ & Diagnóstico clínico & $\begin{array}{l}\text { Sesiones } \\
\text { realizadas } \\
\text { (n) }\end{array}$ & $\begin{array}{c}\text { Inicio de } \\
\text { recuperación } \\
\left(\mathrm{N} .^{\circ} \text { sesión) }\right.\end{array}$ & $\begin{array}{l}\text { Recuperación } \\
\text { final } \\
\text { (día) }\end{array}$ \\
\hline 1 & 0.5 & $\begin{array}{l}\text { Displasia cadera } \mathrm{MP}\left[\mathrm{LD} 87^{\circ} \text { y }\right. \\
\left.\text { LI } 100^{\circ}\right]\end{array}$ & 8 & 4 & 28 \\
\hline 2 & 0.7 & Displasia cadera MPD $96^{\circ}$ & 7 & 2 & 21 \\
\hline 3 & 1 & $\begin{array}{l}\text { Poscirugía }(1 \mathrm{mes}) \text { por } \\
\text { politraumatismo }\end{array}$ & 10 & 3 & 42 \\
\hline 4 & 1 & $\begin{array}{l}\text { Post-cirugía femoral por } \\
\text { traumatismo }\end{array}$ & 11 & 4 & 49 \\
\hline 5 & 4 & $\begin{array}{l}\text { Estenosis vertebral L7 -S1 y } \\
\text { cauda equina }\end{array}$ & 8 & 3 & 28 \\
\hline 6 & 5 & $\begin{array}{l}\text { Displasia cadera [LI } 96^{\circ} \text { y } \\
\text { hernia lumbar L7 - S1 }\end{array}$ & 8 & 2 & 28 \\
\hline 7 & 5 & Displasia de cadera MPI, $100^{\circ}$ & 6 & 3 & 17 \\
\hline 8 & 5 & $\begin{array}{l}\text { Espondiloartrosis T5-T6, T10- } \\
\text { T11 }\end{array}$ & 8 & 2 & 28 \\
\hline 9 & 6 & Hernia protrusiva lumbar & 12 & 4 & 56 \\
\hline 10 & 7 & $\begin{array}{l}\text { Cirugía hemilaminectomia (1 } \\
\text { mes). Condrodistrofia T10, T11 }\end{array}$ & 12 & 5 & 56 \\
\hline 11 & 9 & $\begin{array}{l}\text { Síndrome cauda equina y } \\
\text { artrosis de rodilla }\end{array}$ & 1 & $\mathrm{NR}^{(1)}$ & NR \\
\hline 12 & 9 & $\begin{array}{l}\text { Espondiloartrosis vertebral L7 } \\
-\mathrm{S} 1\end{array}$ & 6 & 2 & 17 \\
\hline 13 & 9 & $\begin{array}{l}\text { Espondiloartrosis deformante } \\
\text { L7 S1, parálisis flácida MP }\end{array}$ & 1 & NR & NR \\
\hline 14 & 12 & $\begin{array}{l}\text { Espondiloartrosis lumbar L7- } \\
\text { S1, Cauda equina }\end{array}$ & 6 & 2 & 17 \\
\hline 15 & 12 & $\begin{array}{l}\text { Artrosis coxofemoral derecha } \\
\text { moderada }\end{array}$ & 1 & NR & NR \\
\hline
\end{tabular}

(1) NR: no respondió al tratamiento

MP: Miembro posterior, LD: Lado derecho; LI: Lado izquierdo; MPD: Miembro posterior derecho; MPI: Miembro posterior izquierdo

retrasando la atrofia muscular, disminuyendo la contractura del tendón y acelerando la recuperación y la funcionalidad, especialmente en animales con lesiones incompletas.
Antes de la terapia, los pacientes presentaban signos de incoordinación motriz, dolor y discapacidad (paraplejia espástica y flácida). Estos signos disminuyeron y no mani- 
Cuadro 3. Efectividad del uso del protocolo de terapia física en caninos con afecciones neurológicas y neuropatías traumáticas, según tipo de diagnóstico clínico de tren posterior

\begin{tabular}{|c|c|c|c|}
\hline Diagnóstico clínico & $\begin{array}{l}\text { Paciente } \\
\left(\mathrm{N} .{ }^{\circ}\right)^{(1)}\end{array}$ & $\begin{array}{c}\text { Pacientes } \\
\text { pre- } \\
\text { fisioterapia } \\
\text { (n) }\end{array}$ & $\begin{array}{l}\text { Recuperados } \\
\text { (n) }\end{array}$ \\
\hline Artrosis moderada coxofemoral derecha & 15 & 1 & 0 \\
\hline $\begin{array}{l}\text { Cirugía hemilaminectomía y } \\
\text { condrodistrofia T10 - T11 }\end{array}$ & 3 & 1 & 1 \\
\hline Displasia cadera & $1,2,6,7$ & 4 & 4 \\
\hline Espondiloartrosis T10- T11, L7- S1 & $8,12,14$ & 3 & 3 \\
\hline $\begin{array}{l}\text { Espondiloartrosis L7-S1. Parálisis } \\
\text { flácida. Infección urinaria y neoplasia } \\
\text { testicular }\end{array}$ & 13 & 1 & 0 \\
\hline $\begin{array}{l}\text { Estenosis vertebral L7-S1 - cauda } \\
\text { equina }\end{array}$ & 5 & 1 & 1 \\
\hline $\begin{array}{l}\text { Estenosis vertebral, artrosis de rodilla, } \\
\text { tumor mamario y lipoma }\end{array}$ & 11 & 1 & 0 \\
\hline Hernia protrusiva lumbar & 9 & 1 & 1 \\
\hline Poscirugía ( 1 mes) por politraumatismo & 3,4 & 2 & 2 \\
\hline Efectividad & & 15 & 12 \\
\hline
\end{tabular}

festaron complicaciones después de la terapia. Si bien la recuperación de un paciente depende del grado de la lesión y del resultado del procedimiento quirúrgico como lo indica Hury (2008), coincide con los resultados negativos de tres pacientes gerontes $(9,9$ y 12 años) en este estudio, debido a que sus lesiones estaban asociadas a otras patologías (tumor testicular, tumor mamario y lipoma), no respondiendo de manera favorable a la terapia efectuada. Por otro lado, las variaciones en la recuperación con respecto al número de sesiones realizadas, se podría explicar por la variación fisiológica de cada individuo y estado de la lesión propiamente dicha.
Todo tratamiento fisioterapéutico depende en gran medida del factor económico, disponibilidad del dueño para aceptar el protocolo fisioterapéutico y asumir la responsabilidad de llevar a la mascota a las sesiones fisioterapéuticas. En este estudio, de los tres pacientes que no respondieron al tratamiento, dos dueños eligieron la eutanasia y solo uno continuó con la recomendación de la acupuntura como terapia alternativa.

Los otros 12 pacientes respondieron positivamente a la terapia física entre la segunda y quinta sesión, pudiendo dejar la medicación de antiinflamatorios y analgésicos 


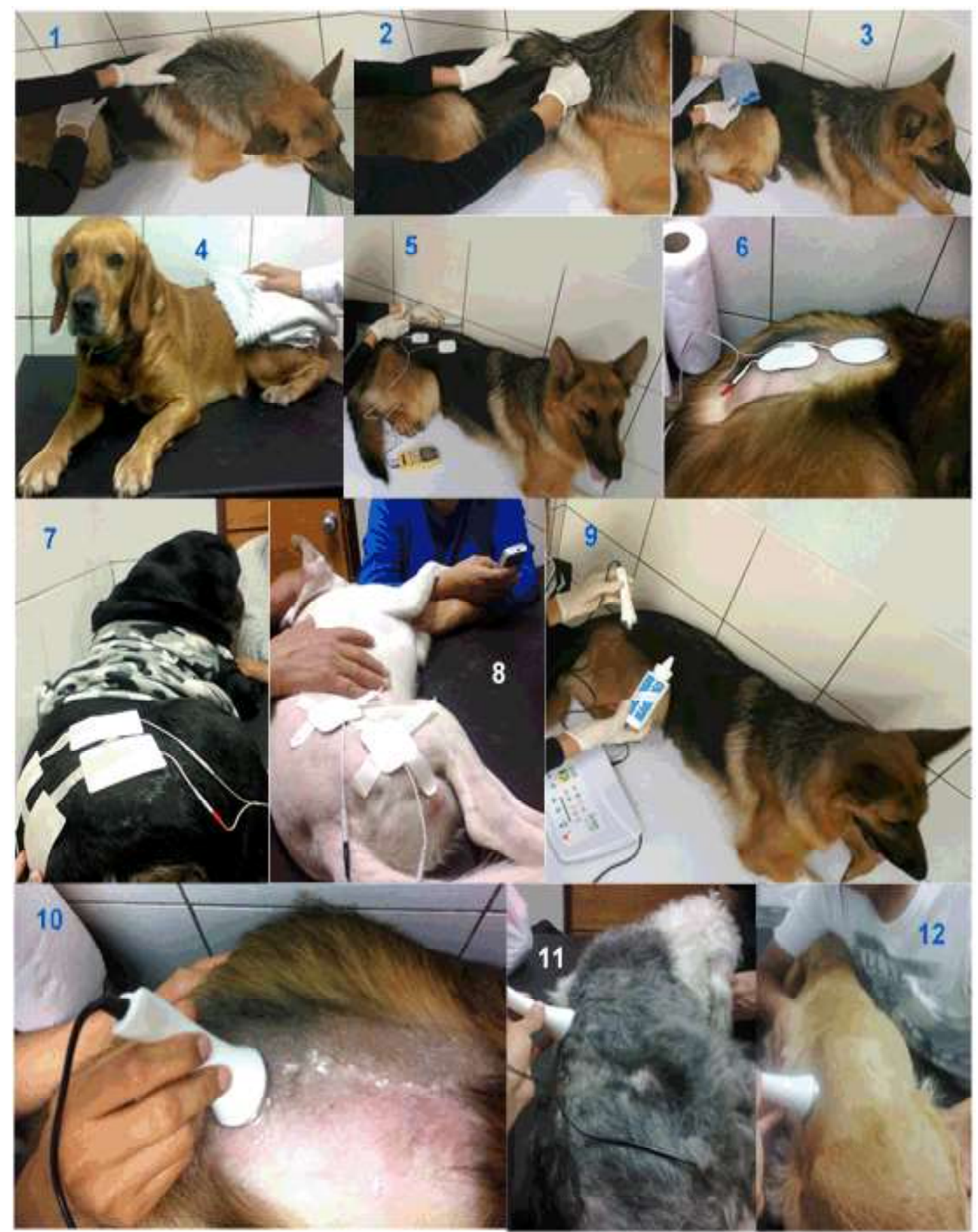

Figura 1. Efecto de tratamiento fisioterapéutico en caninos domésticos con claudicación del tren posterior realizado en la Clínica Veterinaria Cayetano Heredia (Lima, Perú). 1) Masaje stroking. 2) Masaje Kneading. 3) Crioterapia a nivel de la región lumbar. 4) Termoterapia a nivel de la región lumbar. 5) Equipo Electroterapia o TENS. 6) Electroanalgesia en espondiloartrosis lumbar. 7) Electroanalgesia, paciente con diagnóstico displasia de cadera LI $96^{\circ}$ y hernia lumbar L7-S1. 8) Electroestimulación, paciente con atrofia muscular. 9) Equipo de ultrasonido. 10 y 11) Ultrasonido, paciente con diagnóstico espondiloartrosis lumbar. 12) Ultrasonido, paciente con diagnóstico de cauda equina 
convencionales a los pocos días de iniciado el tratamiento fisioterapéutico. Esto debido a que los dueños observaron una disminución de la puntuación del dolor. Si bien las 12 sesiones fisioterapéuticas suelen alarmar al dueño, es importante la información detallada del procedimiento a fin de conseguir la concientización y cooperación del dueño. En este estudio, se pudo observar el esfuerzo por parte de los dueños para asistir a las sesiones; aunque además, es importante indicar que la terapia fue proporcionada en forma gratuita, al tratarse de un estudio de investigación.

El beneficio de los masajes en el estudio de Kong et al. (2013), demostró efectos inmediatos aliviando el dolor de hombro y cuello en pacientes humanos, de allí que si esta técnica se combina con equipos y medios físicos (crioterapia, termoterapia, TENS y ultrasonido), puede generar buenos resultados.

De los pacientes derivados a fisioterapia, es importante la comunicación con el médico tratante, especialmente si son pacientes posquirúrgicos, a fin de conocer el grado de evaluación neurológica previo a una cirugía y los tratamientos convencionales realizados para establecer el pronóstico de la fisioterapia (L. Carpio, Lima, Comunicación personal). Los propietarios de los canes deben ser informados que se puede requerir hasta 12 sesiones para observar la respuesta, y que se debe continuar hasta obtener un adecuado rendimiento (Calzada, 2011), tomando en consideración el protocolo de trabajo (Hury, 2008). Durante todas las sesiones se resguardó la integridad del paciente, especialmente al usar las compresas frías y calientes para evitar quemaduras. Asimismo, en la electroterapia se debe tener especial cuidado con la intensidad y frecuencia en relación al tipo de lesión (Shearer y August, 2017).

Hanks et al. (2015) y Shearer et al. (2017) mencionan que la fisioterapia en animales de compañía está teniendo, a nivel glo- bal, una mayor consideración dada la necesidad de disminuir los procesos dolorosos, conllevando al restablecimiento de la biomecánica de los músculos y mejorando la calidad de vida de los pacientes.

\section{Conclusiones}

El uso del protocolo de terapia física (masajes, termoterapia, electroterapia y ultrasonido) en los caninos con problemas de tren posterior como resultado de afecciones neurológicas y neuropatías traumáticas resultó efectivo en el $80 \%$ de los pacientes.

\section{Literatura Citada}

1. Calzada A. 2011. Rehabilitación en un perro con fractura vertebral sin resolución quirúrgica. Rev Argos 126: 66.

2. Capote A, López Y, Bravo T. 2009. Agentes físicos. La Habana, Cuba: Ciencias Médicas. $325 \mathrm{p}$.

3. Correa F. 2006. Fisioterapia veterinária. Curitiba, Brasil: Facultad de Ciéncias Biológicas e da Saúde, Universidade Tuiuti do Paraná. 79 p.

4. Colorado State University. 2006. Canine acute pain scale. Veterinary Medical Center [Internet]. Available in: https://www.polyu.edu.hk/ro/forms/ Attachment\%201_Colorado\%20State\%20U\%20-Pain\%20Scale.pdf

5. Dávila R, Fernández V, Chavera A, Díaz D. 2002. Frecuencia de displasia de cadera en caninos Rottweiler mayores de un año de edad. Rev Inv Vet Perú 13:32-37.

6. Del Pueyo G 2011. Fisioterapia y rehabilitación veterinaria. España: Servet. $184 \mathrm{p}$.

7. Hanks J, Levine D, Bockstahler B. 2015. Physical agent modalities in physical therapy and rehabilitation of small animals. Vet Clin North Am Small Anim Pract 45: 29-44. doi: 10.1016/ j.cvsm.2014.09.002 
8. Hury L. 2008. Presentación de un caso clínico de displasia de cadera. Rev Argos 102: 48-50.

9. Jaramillo-Antillón J. 2005. Historia y filosofía de la medicina. Costa Rica: Ed Universidad de Costa Rica. 401 p.

10. Kassai T. 1998. Helmintología veterinaria. Zaragoza, España: Acribia. 296 p.

11. Kong L, Zhan H, Cheng Y, Yuan W, Chen B, Fang M. 2013. Massage therapy for neck and shoulder pain: a systematic review and meta-analysis. Evidence-B Compl Alternative Med ID 613279. doi: 10.1155/2013/613279

12. Levine D, Mills DL, Mynatt T. 2001. Effects of 3.3-MHz ultrasound on caudal thigh muscle temperature in dogs. Vet surgery 30: 170-174.

13. Lindley $S$, Watson P. 2015. Manual de rehabilitación y cuidados paliativos en pequeños animales. UK: Lexus BSAVA. $532 \mathrm{p}$.

14. McGonagle L, Blythe L, Levine D. 2004. History of canine physical rehabilitation. In: Canine rehabilitation \& physical therapy. USA: Elsevier. p 1-4.

15. Millis D, Levine D. 2013. Canine rehabilitation and physical therapy. $2^{\text {nd }} \mathrm{ed}$. USA: Elsevier. $784 \mathrm{p}$

16. Miró-Rodríguez F, Cond-Ruiz C, Martínez-Galisteo A. 2007. La fisioterapia: un medio afectivo en el tratamiento conservador de la osteoartritis de rodilla del perro. RECVET 2(7). [Internet]. Disponible en: http://www.veterina- ria.org/revistas/recvet/n070707/ 070701.pdf

17. Nelson R, Couto G. 2010. Medicina interna de pequeños animales. $4^{\mathrm{a}}$ ed. Barcelona, España: Elsevier. 1504 p.

18. Oliver J, Lorenz M, Kornegay J. 2003. Manual de neurología veterinaria. $3^{\mathrm{a}} \mathrm{ed}$. Barcelona, España: Multimédica. 484 p.

19. Ortega A. 2013. Fisioterapia como tratamiento alternativo en compresión de cauda equina. Ortovet, España. [Internet]. Disponible en: http://www.ortocanis.com/es/content/78-fisioterapiacomo-tratamiento-alternativo-encompresion-de-cauda-equina

20. Shearer T, August K. 2017. Physical medicine, rehabilitation, and complementary and integrative medicine treatment modalities. In: Shanan A, Shearer T, Pierce J (eds). Hospice and palliative care for companion animals: principles and practice. EEUU: Wiley Blackwell.p 181-198.

21. Sterin G 2008. ¿Terapia física en medicina felina? Argos. España. [Internet]. Disponible en: http://argos.portalveterinaria.com/noticia/1476/artculosarchivo/terapia-fisica-en-medicinafelina.html

22. Van Dyke J. 2009. Canine rehabilitation: an inside look at a fast-growing market segment. DVM360. [Internet]. Disponible en: http://veterinarynews.-dvm360. com/canine-rehabilitation-inside-lookfast-growing-market-segment?rel= canonical 